\title{
Arbeits- und prozessorientierte Digitalisierung
}

\section{Vorgehensweisen, Praxiserfahrungen und Erkenntnisse}

\author{
Gabriele Held, Beate Schlink, Jörg Bahlow, Wolfgang Kötter, \\ Sebastian Roth, Alexander Bendel, Erich Latniak, Frank Lennings und \\ Sebastian Terstegen
}

\subsection{Unser Ziel: Digitalisierungspotenziale nutzen - Arbeitsaufgaben optimal unterstützen}

In der praktischen betrieblichen Umsetzung gelingt es häufig nur unter großer Anstrengung, IT-Einführungsprozesse mit den arbeitsprozessbezogenen Bedarfen der Nutzenden in Einklang zu bringen. Oft entstehen „bereichsoptimale“ Lösungen, die durch arbeitsgestalterische Kompromisse in vor- und nachgelagerten Prozessschritten erkauft werden müssen. Zudem bleibt oft eine Steuerungskomplexität von den Beschäftigten zu bewältigen, die dabei gegebenenfalls auch „am System vorbei“ arbeiten müssen [5]. Dadurch entsteht ein erheblicher, systembegleitender Regelungsund Kommunikationsbedarf, der zu Reibungsverlusten und Konflikten führen kann. Es kommt zu Medienbrüchen, Zusatzaufwand, Informationsverlusten und unnötigen Konflikten. Unternehmen und Beschäftigte erleben oft, dass der erwartete Nutzen digitaler Lösungen nicht eintritt, weil technische Lösungen uneinheitlich umgesetzt beziehungsweise gehandhabt werden und im laufenden Betrieb viele Änderungen

G. Held $\cdot$ B. Schlink $(\bowtie)$

Fachbereich Fachkräftesicherung, RKW Rationalisierungs- und Innovationszentrum der

Deutschen Wirtschaft e. V. Kompetenzzentrum, Eschborn, Deutschland

J. Bahlow $\cdot$ W. Kötter $\cdot$ S. Roth

GITTA Gesellschaft für interdisziplinäre Technikforschung Technologieberatung

Arbeitsgestaltung mbH, Berlin, Deutschland

A. Bendel · E. Latniak

Institut Arbeit und Qualifikation Universität Duisburg-Essen, Duisburg, Deutschland

F. Lennings $\cdot S$. Terstegen

ifaa - Institut für angewandte Arbeitswissenschaft e. V., Fachbereich Unternehmensexzellenz,

Düsseldorf, Deutschland 
gleichzeitig und wenig abgestimmt stattfinden. Zudem leiden die betrieblichen Digitalisierungsprozesse immer wieder unter knapper Kapazität beim Einsatz fachlicher und technischer Spezialisten.

Im Forschungs- und Entwicklungsprojekt „APRODI - Arbeits- und prozessorientierte Digitalisierung in Industrieunternehmen" nahmen wir dies zum Anlass, neue Wege der Gestaltung von betrieblichen Informationsräumen und IT-unterstützten Arbeitssystemen zu erproben. Dabei sollten die jeweilige Ausgangssituation und Kultur des Unternehmens, vorhandene und bewährte technische Systeme und die Voraussetzungen, Möglichkeiten und Interessen der Mitarbeitenden gleichermaßen berücksichtigt werden. Die Grundsätze der Ganzheitlichkeit, der Integration und vor allem der Partizipation galten als wichtige Erfolgsfaktoren. An soziotechnischen Konzepten orientierte Vorgehensweisen sollten sicherstellen, dass das Zusammenspiel von Mensch, Technik und Organisation in den Fokus rückt.

Handlungsleitend für unseren Forschungsverbund waren die Fragen:

1. Wie kann ein soziotechnischer Digitalisierungsprozess auf Grundlage der betrieblichen und individuellen Voraussetzungen möglichst optimal für Betrieb und Beschäftigte gestaltet werden? Welche hemmenden und fördernden Faktoren sind dabei zu bearbeiten? Wie lassen sich vorhandene Potenziale erschließen und Risiken möglichst frühzeitig ermitteln und verhindern?

2. Wie können solche betrieblichen soziotechnischen Einführungsprozesse mit geeigneten Instrumenten und Hilfsmitteln unterstützt werden? Welche Qualifizierungs- und Unterstützungsmaßnahmen haben sich bewährt?

3. Wie kann die Beteiligung der Beschäftigten in diesen Prozessen gestaltet werden, um eine möglichst gute arbeitsplatznahe technische und organisatorische Unterstützung der Arbeitsprozesse zu gewährleisten?

Antworten hierzu lieferten die Arbeiten in fünf Unternehmen, in denen verschiedene betriebliche Digitalisierungsprozesse intensiv begleitet, Vorgehensweisen und Methoden entwickelt, angewendet und deren Wirkung evaluiert wurden. Der kontinuierlich verbundintern stattfindende Austausch und begleitende Diskussionen mit Sozialpartnern und in Fachkreisen unterstützten einerseits den Lernprozess der Verbundpartner und ermöglichten es andererseits, erfolgsrelevante und übertragbare Faktoren zu formulieren, um sie für Wissenschaft und Praxis nutzbar zu machen.

\subsection{Der soziotechnische Ansatz in Digitalisierungsprozessen}

Im APRODI-Projekt knüpften wir an ein soziotechnisches Grundverständnis für die Arbeits- und Systemgestaltung in Digitalisierungsprozessen an, soweit das in den betrieblichen Projekten möglich war. Soziotechnische (ST) Gestaltungsansätze werden zunehmend für aktuelle Digitalisierungsprozesse wiederentdeckt. Nach unserem Ver- 
ständnis vermitteln ST-Konzepte Orientierungswissen für die betrieblich Handelnden bei der Arbeits- und Organisationsgestaltung in Digitalisierungsprozessen; sie bieten durch Prinzipien, Modelle und Vorgehensweisen eine Art „Landkarte“ für unbekanntes Gelände - die zukünftige Organisation der digitalisierten Arbeit - und helfen den Akteuren, diese Organisation zu entwickeln [1]. Dabei bieten ST-Ansätze den Vorteil, dass sie die für die Digitalisierung relevanten Aspekte „Mensch“, „Technik“ und „Organisation“ im Zusammenhang berücksichtigen. Ziel ist eine integrierte Verbesserung dieser drei Aspekte, die durch Beteiligung der Nutzenden im Gestaltungsprozess ermöglicht wird.

Neu an den aktuellen Digitalisierungsprozessen ist, dass sich ein großer Teil der Kommunikation zwischen den an der Produktion oder Dienstleistungserstellung Beteiligten über unterschiedlichste digitale Medien abspielt. Dies ermöglicht neben einer höheren Geschwindigkeit auch die zeitliche und räumliche Entkoppelung von Tätigkeiten, die früher notwendig an einem Ort stattfanden. Dabei sind technische Infrastruktur und Ausrüstung vielfach nicht mehr vom Arbeitsprozess trennbar. Andererseits überschreitet die notwendige Kommunikation häufig die räumlichen und rechtlichen Grenzen des Betriebs; die Informationssysteme sind zunehmend unternehmensweit oder -übergreifend im Einsatz, häufig ineinander verschachtelt und entwickeln sich in unterschiedlichen Geschwindigkeiten weiter.

Wir sprechen deshalb im APRODI-Kontext von zu gestaltenden ,Systemen von Arbeits- und Informationssystemen“. Damit schließen wir an neuere Ansätze in der STDiskussion an, die sich um die Vorstellung eines „eco-systems“ [6] drehen. Gemeint ist damit die Unternehmensgrenzen überschreitende Umgebung des Produktionsprozesses mit internen wie externen Kunden, Zulieferern, Dienstleistern und weiteren Kreisen, die die Arbeits- und Informationssysteme nutzen und die in die Gestaltung eingebunden werden sollten. Vor diesem Hintergrund stand in allen betrieblichen Projekten die Frage zur Beantwortung an, wer wann und in welcher Form in die Digitalisierungsprozesse einzubinden und zu beteiligen ist. Hierauf wurden jeweils angepasste Lösungen gefunden.

Zudem gingen wir zu Beginn von APRODI von einem gemeinsamen Prozessverständnis aus, das eine Vorstellung vermittelte, welche Schritte in einem betrieblichen Digitalisierungsprozess durchlaufen werden (Abb. 2.1). Unsere gemeinsame Grundorientierung war es, dass Digitalisierung nicht einem linearen Ablauf fester Schritte folgt, sondern in einem zyklischen Vorgehen mit Reflexions- und Rückkoppelungsschleifen verläuft. Dies versetzt die Beteiligten in die Lage, auf wechselnde betriebliche Bedingungen adäquat reagieren zu können. Auch hier schließen wir an aktuelle Konzepte der angelsächsischen ST-Diskussion an, die von ähnlichen Prozessmodellen ausgehen [9]. 


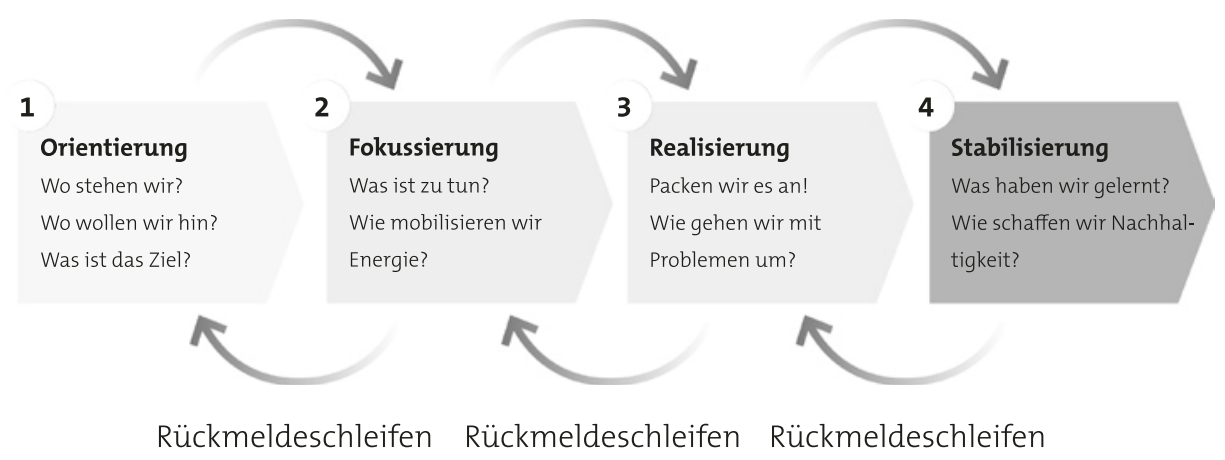

Abb. 2.1 Phasen des Ablaufs in den betrieblichen Teilprojekten, in Anlehnung an [4]

\subsection{Erfahrungen aus den APRODI-Betriebsprojekten}

In der dreijährigen Projektlaufzeit haben wir uns in fünf APRODI-Unternehmen mit unterschiedlichen Aspekten der Digitalisierung auseinandergesetzt. Wir beschreiben im Folgenden einige für die Veränderungsphasen prägende Vorgehensweisen und Methoden und welche Erfahrungen damit in einzelnen Betriebsprojekten gemacht wurden.

\subsubsection{Orientierungsphase: Vorgehen am Beispiel der ZF Friedrichshafen AG, Schweinfurt}

Unabhängig von Themen und Inhalten steht jedes betriebliche Digitalisierungsprojekt vor der Herausforderung, von der Vielfalt der Perspektiven und Möglichkeiten zu einem zielgerichteten und koordinierten Digitalisierungsprozess zu gelangen. Am Beispiel der ZF Friedrichshafen AG beschreiben wir ein typisches, vom Betriebspartner als zielführend bewertetes Vorgehen in der Orientierungsphase.

Die ZF Friedrichshafen AG ist ein weltweit führender Technologiekonzern in der Antriebs- und Fahrwerktechnik sowie der aktiven und passiven Sicherheitstechnik mit weltweit ca. 150.000 Beschäftigten. Am Standort Schweinfurt haben die 300 Beschäftigten der zentralen Instandhaltung im Wesentlichen die Aufgabe, die technische Verfügbarkeit der Produktions-Maschinen und -Anlagen sicherzustellen. Rund 270 Beschäftigte sorgen in der zentralen Logistik für die Warenströme von Komponenten, Fertigwaren und Verpackungen sowie deren Lagerung. Im Zuge von Prozesskettenoptimierungen war es in den letzten Jahren auch um die zunehmende Vernetzung und Digitalisierung der Geschäftsprozesse sowie der Kommunikation insgesamt gegangen. Aufgrund wachsender Flexibilitätsanforderungen und der daraus resultierenden Erhöhung der Reaktionsgeschwindigkeit nahm der Kommunikationsaufwand zwischen allen Prozess-Partnern ständig zu; viele Ressourcen in den beteiligten 
Fachbereichen wurden dadurch gebunden. Vor diesem Hintergrund wollte die ZF Friedrichshafen $\mathrm{AG}$ in diesen Fachbereichen Medienbrüche reduzieren und die prozessübergreifende Kommunikation und Zusammenarbeit intern/extern durch erweiterte Kompetenz und Nutzung digitaler Medien verbessern. Immer wieder wurde auch die selbstentwickelte Software, die für die Lagerverwaltung mit genutzt wird, diskutiert. Das Access-basierte digitale Assistenzsystem „Instandhaltungsplanungssystem IPS“ sollte aufgrund der umfassenden Akzeptanz bei den Nutzern weiterentwickelt werden.

Ein Promotoren-Workshop zu Beginn des APRODI-Projekts diente dazu, die Sichtweisen und Zielvorstellungen der wichtigsten ZF-internen Projektbeteiligten und Projekt-,,Treiber“(Promotoren) mit den Sichtweisen und Vorschlägen der externen APRODI-Forschungspartner abzugleichen und auf dieser Grundlage die Orientierungsphase zu planen: Das Gesamtziel bestand darin, die Ersatzteilbelieferung der Instandhaltung mit Logistik und Einkauf zu unterstützen. Das oberste Ziel war vor diesem Hintergrund relativ einfach $\mathrm{zu}$ benennen, aber umso schwieriger umzusetzen: Das richtige Teil, zur richtigen Zeit, am richtigen Ort und nach Möglichkeit noch bei optimalen, das heißt niedrigen Beständen. Die Zielklärung mit der Geschäftsleitung brachte eine hohe Übereinstimmung im Hinblick auf diese Herausforderungen zu erzielen. Es war klar, dass nicht nur die durch das APRODI-Projekt mögliche externe Unterstützung aktiv genutzt, sondern auch die nötigen internen Ressourcen für das Projekt mobilisiert werden mussten.

Ein Workshop mit Führungskräften aus den oberen Managementebenen stellte die inhaltliche und thematische Ausrichtung des Projekts auf den Prüfstand. Nach der Standortbestimmung im Führungskreis erfolgte ein „Visionscoaching“ zur Einordnung der Projektziele in eine längerfristige Perspektive zur Entwicklung des Standorts. Im Anschluss an eine Meilenstein-Planung erfolgte die Bildung des Projektteams zur operativen Durchführung des Projekts sowie eines Steuerkreises, der in regelmäßigen Abständen - insbesondere zu Projektmeilensteinen - Berichte entgegennehmen und strategische Projektentscheidungen treffen sollte.

Zur Analyse der Ausgangssituation wurden vor allem Beobachtungsinterviews mit den Instandhaltungsfachkräften einer Schicht, mehrere aufeinanderfolgende Expertenworkshops mit Führungskräften, Selbstaufschreibungen des Instandhaltungspersonals und Betriebsrundgänge genutzt.

Die Auswertungen dieser Aktivitäten durch das APRODI-Projektteam zeigten viele - zu einem erheblichen Teil prozessbezogene, untereinander vernetzte und nur indirekt digitalisierungsrelevante - „Baustellen“ auf. Verbesserungs- und Umsetzungsvorschläge wurden gemeinsam erarbeitet und Vorlagen mit Hintergrunddaten, Aufwands- und Nutzenbetrachtungen und Entscheidungsoptionen für den Steuerkreis in einer Projektteam-Klausur erstellt. In einem anschließenden Fokusentscheid konnte der Steuerkreis aufgrund der Vorlagen konkrete Maßnahmen genehmigen und die wesentlichen Handlungsfelder bestimmen: Ersatzteilfestlegung, Ersatzteilbeschaffung sowie Ersatzteilhandling (Lagerung und Andienung). Außerdem wurde die Erarbeitung einer Ersatzteilmanagementstrategie beschlossen. Dieser Schritt stellte den ersten Meilenstein 
im betrieblichen APRODI-Teilprojekt dar - und zwar nicht nur im Hinblick auf die Zielklärung und Maßnahmenplanung, sondern auch auf die Einbeziehung aller Stakeholder, die Etablierung einer funktionsfähigen Projektstruktur und die Akzeptanz der APRODIAktivitäten bei den Führungskräften.

\subsubsection{Fokussierungsphase: Soziotechnisches Lastenheft - Nutzeranforderungen aufnehmen (Agfa-Gevaert HealthCare GmbH)}

Die Agfa-Gevaert HealthCare GmbH stellt im Werk Peißenberg mit circa 300 Beschäftigten hochmoderne Digitizer und Printer sowie Direktradiologie-Systeme für Röntgenbilder her. Die vielstufige Montage dieser komplexen Medizingeräte, die Teilevielfalt sowie regulatorische Anforderungen machen genaueste Beschreibungen der Arbeitsabläufe notwendig. Bisher waren alle Informationen, die ein Werker für die Gerätemontage braucht, in einem Aktenordner abgelegt: Montageschritte, Stücklisten, Drehmomenttabellen und so weiter. Sie stellten eine hilfreiche Informationsquelle bei Anlernvorgängen dar, boten aber aufgrund der umständlichen Handhabung zwar eine normkonforme jedoch keine so effiziente Unterstützung im Montagealltag.

Agfa hatte deshalb das Ziel, ein digitales Assistenzsystem zur Unterstützung der Beschäftigten in der Montage zu erarbeiten. Das APRODI-Betriebsteam wählte bei der Anforderungsermittlung zur Entwicklung des Systems einen besonderen Weg.

Während bisherige Konzepte zur Anforderungsermittlung umfangreiche Kriterien „guter" Software (,Requirements Specifications“) und vor allem eine vollständige Anforderungsliste vor Beginn der Softwareentwicklung erforderten, entschieden sich die Verantwortlichen im Projekt für ein anderes, ganzheitliches und partizipatives Vorgehen. Denn es wurde bereits im Vorfeld das Risiko gesehen, dass Softwareentwickler technikgetrieben und an Vollständigkeit orientiert an die Anforderungsermittlung herangehen würden. Stattdessen wollte man sich nicht ausschließlich auf Expertenwissen verlassen, sondern die Bedürfnisse und Bedarfe der Werker vor Ort einbeziehen, die anschließend mit dem Assistenzsystem arbeiten sollten. Anforderungen, die sich bei der Testung des Systems vor Ort ergeben oder ändern, sollten ebenso im Entwicklungsprozess berücksichtigt werden.

Für die Umsetzung wurden in einer Orientierungsphase vier halbtägige Beobachtungsinterviews mit den Werkern vor Ort durchgeführt. Neben der Dokumentation der Arbeitstätigkeit, der Abläufe, Aufgaben und Bedingungen am Arbeitsplatz wurden die Stärken und Schwächen der derzeitigen Unterstützungslösung sowie Potenziale und Risiken eines digitalen Assistenzsystems bei den Beschäftigten erfragt. Aus den Ergebnissen hat das APRODI-Betriebsteam in der Fokussierungsphase Anforderungen abgeleitet und in einem soziotechnischen Lastenheft dokumentiert. Soziotechnische Lastenhefte bauen auf den Grundgedanken des soziotechnischen Systems auf [8] (siehe auch Abschn. 2.2). Sie beinhalten neben den technischen Voraus- 
setzungen, Zielsetzungen und Rahmenbedingungen, die bei der Entwicklung des angestrebten Assistenzsystems zu berücksichtigen sind, auch Anforderungen, die sich aus dem sozialen Teilsystem ergeben und zum Beispiel die sozialen Vorbedingungen und Auswirkungen der Systementwicklung in den Blick nehmen. Vorerfahrungen, Expertenwissen, Anforderungen und Wünsche der zukünftigen Systemnutzer wurden mit aufgenommen. Dabei ist ein Anforderungskatalog entstanden, der regulatorische, aufgabenbezogene, organisationsbezogene und technikbezogene Aspekte umfasst. Ein Beispiel dafür ist die Verknüpfung von Berechtigungsabfragen (Ist der aktuelle Nutzer aufgrund seines Trainingsstatus berechtigt, die Baugruppe aus der Montageanweisung zu montieren?) mit einer Qualifikationsmatrix (Wer kann was? Wie muss wann qualifiziert werden?) und zentralen Nutzerkennungen (= Login-Daten). Im Lastenheft wurden die Anforderungen an die zentralen Eigenschaften des einzusetzenden Assistenzsystems definiert. Zum Beispiel wurde - neben der situations- und qualifikationsangemessenen Führung des Werkers durch den Montageablauf - die Möglichkeit der Rückmeldung nicht montagegerecht geplanter Arbeitsschritte an die Konstruktionsabteilung sowie die individuelle Dokumentation von Unregelmäßigkeiten berücksichtigt.

Das soziotechnische Lastenheft erwies sich als eine solide Grundlage zur Auswahl von IT-Dienstleistern, die sich mit der vom APRODI-Betriebsteam gewünschten Vorgehensweise identifizieren können. Letztlich wurden vier aussichtsreiche Anbieter zu einer Präsentation ihrer Umsetzungslösung nach Peißenberg eingeladen. Zum Zeitpunkt der Berichterstattung befand sich Agfa in der Beauftragung eines IT-Dienstleisters zur Entwicklung des Montage-Assistenzsystems.

\subsubsection{Fokussierungsphase: Das Digitalisierungs-Reifegradmodell als individuelles Analysewerkzeug für die Bewertung und Steuerung betrieblicher Digitalisierungsprozesse gestalten (Continental Teves)}

Im Frankfurter Werk der Continental Teves AG \& Co. oHG werden elektronische Bremssysteme hergestellt. Der Automatisierungsgrad in der Produktion beträgt für die meisten Produkte $99 \%$. Ein Ziel des Unternehmens im Rahmen des APRODI-Projekts ist, den Einsatz der Digitalisierung im Werk zu erweitern, um die prozessübergreifende Kommunikation und Zusammenarbeit durch Systemgestaltung und Kompetenzentwicklung weiter zu verbessern. Das Managementteam erarbeitete eine Vision „Werk Frankfurt 2025“, die verschiedene Handlungsfelder zur weiteren Verbesserung und störungsfreien Aufrechterhaltung des bereits jetzt weitgehend automatisierten Materialflusses umfasst. Kompetenzmanagement und Beteiligung der Mitarbeiter sind darin ebenso bedeutsam wie Digitalisierungsmaßnahmen.

Zur Konkretisierung von Maßnahmen in diesen Handlungsfeldern sowie zur Verbesserung der Information und Beteiligung der Mitarbeiter sollte ein unternehmensspezifisches Reifegradmodell dienen. Dieses wurde im Rahmen eines Workshops erarbeitet, 
an dem sowohl die Mitglieder des Managementteams als auch zukünftige Führungskräfte teilnahmen. Die Modellentwicklung umfasste die Arbeitsschritte: 1. Erfolgsfaktoren ,guter“ Digitalisierung sammeln und strukturieren, 2. Bewertungskriterien zu den Erfolgsfaktoren ableiten, 3. Bewertungsfragen formulieren, 4. geeignete Skalierung entwickeln und 5. Modell erproben.

Für die Umsetzung der ersten vier Schritte benötigte das Team knapp anderthalb Tage. Das resultierende Modell umfasst die Bewertungskriterien Change Management, Kommunikation und Information, Führung und Zusammenarbeit, Kompetenzen, Kultur und Mindset, Technologie Hardware, Technologie Software sowie Gestaltungsspielräume, Räume und Ressourcen. Diese Kriterien haben alle Teilnehmer im ersten Schritt gemeinsam erarbeitet. Im zweiten Schritt konkretisierten die Teilnehmer die Kriterien und operationalisierten sie für eine Bewertung. Dazu formulierten sie vertiefende Bewertungsfragen bzw. Aussagen beispielsweise zum Kriterium „Kompetenzen, Kultur und Mindset“: „Die zukünftig notwendigen Kompetenzen für die Digitalisierung sind bekannt.“ oder „Ein Soll-Ist-Vergleich ist erfolgt.“ Den Aussagen können die Bewertungen „nicht erkennbar“ (0), „teilweise“ (1), „überwiegend“ (2) und „,in vollem Umfang“ (3) zugeordnet werden. Der Reifegrad eines Kriteriums entspricht der Summe der Punkte aller abgegebenen Aussagen im Verhältnis zur maximal möglichen Punktzahl.

Das Reifegradmodell wurde von 40 Personen aus allen Bereichen des Werkes erprobt, um Gestaltungsfelder mit hohem Handlungsbedarf zu identifizieren. Der Personenkreis war zudem hierarchieübergreifend zusammengesetzt. Die Ergebnisse wurden im Managementteam vorgestellt und diskutiert, mögliche Handlungsfelder und Maßnahmen gesammelt und erörtert und abschließend für die Umsetzung festgelegt. Diese umfassten u. a. die Themen Kompetenzmanagement sowie den wirtschaftlichen und beteiligungsorientierten Einsatz von Human Machine Interfaces (HMI) in Produktion, Logistik und Instandhaltung.

Ein betriebsspezifisches Reifegradmodell kann mit geringem Aufwand erstellt werden und sowohl die Orientierungs- und Fokussierungs- als auch die Umsetzungs- und Stabilisierungsphase unterstützen. Es ist geeignet, unternehmensspezifische strategische Aspekte zu konkretisieren und den Ist-Zustand hierzu beteiligungsorientiert zu erfassen. Dies kann die Motivation der Beteiligten, deren Identifikation mit dem Projekt sowie die Akzeptanz für abgeleitete Maßnahmen erhöhen. Über die hier beschriebene erste Anwendung hinaus bietet ein unternehmensspezifisches Reifegradmodell noch weitere Anwendungsmöglichkeiten, beispielsweise den Kreis der Antwortenden gezielt zu erweitern, um Ergebnisse hierarchie- und bereichsspezifisch auszuwerten und entsprechende Maßnahmen abzuleiten. Darüber hinaus können Befragungen in regelmäßigen Abständen wiederholt werden, um die Wirkung umgesetzter Maßnahmen zu kontrollieren. Sind erwartete und vereinbarte Ziele erreicht, können schrittweise neue Handlungsschwerpunkte und Maßnahmen in den Blick genommen werden. Dies ermöglicht einen effektiven und effizienten Ressourceneinsatz in Digitalisierungsprojekten. 


\subsubsection{Realisierungsphase: Software-Entwicklung mit den Nutzern bei DuBay}

Die DuBay Polymer GmbH ist ein Joint Venture der Konzerne LANXESS und DuPont de Nemours. Das Unternehmen produziert in Hamm-Uentrop pro Jahr bis zu $80.000 \mathrm{t}$ PBT-Polymere (Polybutylenterephthalat). DuBay Polymer verfolgte von Anfang an ein weitreichendes Teamkonzept, nach dem alle gut 100 Mitarbeiter auch am Management und der Organisation des Unternehmens mitwirken. DuBay hat entsprechend eine Organisationsstruktur geschaffen, bei der jeder Mitarbeiter sowohl einen funktionalen als auch einen administrativen Aufgabenbereich übernimmt. So überwacht etwa ein Schichtwerker die Anlage am Leitstand; ebenso kümmert er sich um die Planung eines Projekts, organisiert die Urlaubsplanung oder sorgt bei Krankheitsausfällen für Vertretung.

Diese Organisationsstruktur erfordert vielfältige Kommunikationsprozesse, um alle notwendigen Abstimmungen $\mathrm{zu}$ gewährleisten. $\mathrm{Zu}$ deren Unterstützung wurde im Rahmen des APRODI-Projekts eine digitale Kommunikations- und Informationsplattform aufgebaut mit dem Ziel, einerseits Funktionen mehrerer genutzter Softwareprogramme zu bündeln und andererseits erkannte Schwachstellen (wie etwa redundante Datenhaltung, Unübersichtlichkeit) zu beheben. So sollen Zusatzaufwände, Unterbrechungen bei der Arbeit etc. reduziert werden. Zudem sollte das System individuell an und durch die Nutzer angepasst werden können. Insgesamt versprach sich das Management durch diesen Schritt eine Förderung der Beteiligung im Unternehmen, zum Beispiel über die Einrichtung von Foren, in denen spezifisches Erfahrungswissen nutzbar gemacht werden kann.

Die Aufgabe bestand darin, die Kommunikationsplattform im Sinne der Nutzeranforderungen zu gestalten. Hierfür wurde bei DuBay ein Vorgehensmodell nach Winby und Mohrman (2018) genutzt, das für die digitale soziotechnische Systemgestaltung entwickelt wurde. Das Modell lässt sich in vier Phasen unterteilen: Analysephase, Designphase, Test- und Lernphase sowie Skalierungsphase. In der Analysephase wurden Teambesprechungen begleitet, Beobachtungsinterviews durchgeführt und im Rahmen von Workshops mit den Beschäftigten und dem Management die Kommunikationsprozesse untersucht („Mapping“). Aus gemeinsam entwickelten Alternativen (,Varianzanalyse") konnten konkrete Anforderungen für das zu gestaltende IT-System abgeleitet werden. Zur Umsetzung dieser Anforderungen wurde eine anpassbare Standard-Software ausgewählt, die bereits für das Dokumentenmanagement eingesetzt wurde, aber darüber hinaus auch die kollaborativen Funktionalitäten bereitstellt.

Die in den Workshops erarbeiteten Systemanforderungen wurden von den internen ITExperten in Prototypen umgesetzt, die mithilfe soziotechnischer Heuristiken [3] bewertet und angepasst wurden. Bei den Heuristiken handelt es sich um acht Beurteilungskriterien oder ,Daumenregeln“, mit deren Hilfe sich Verbesserungspotenziale von Arbeitssystemen identifizieren lassen. Mit ihrem Einsatz wurde bereits in der Designphase eine ganzheitliche und nutzerzentrierte Perspektive in der Gestaltung berücksichtigt. Funktionsfähige Prototypen kommen im Rahmen einer Test- und Lernphase bereits in ausgewählten 
Pilotbereichen zum Einsatz. Erfahrungen und Berichte von Anwenderinnen und Anwendern fließen in die weitere Entwicklung der Plattform ein und führen bei Bedarf zu Anpassungen.

Sowohl das eingesetzte Vorgehensmodell als auch die verwendeten Kriterien haben sich bisher als adäquate soziotechnische Gestaltungsinstrumente erwiesen und hilfreiche Orientierung im Digitalisierungsprozess geboten. Planung und Umsetzung des eingeführten Informations- und Kommunikationssystems erfolgten partizipativ und ganzheitlich mit Blick auf die jeweiligen Arbeitsprozesse. Adressatengerecht angepasst sind beide Instrumente besonders geeignet, um von betrieblichen Akteuren effektiv eingesetzt zu werden und zu einer ganzheitlichen und gesundheitsförderlicheren Systemgestaltung beizutragen.

\subsubsection{Realisierungsphase: Azubis bauen digitale Kompetenzen im Shopfloor Management auf (John Deere)}

Die John Deere GmbH \& Co. KG beschäftigt rund 6.400 Mitarbeiter an sieben Standorten in der Bundesrepublik Deutschland. Mit rund 2.800 Beschäftigten ist das John Deere Werk Mannheim seit mehr als 42 Jahren Deutschlands größter Hersteller und Exporteur landwirtschaftlicher Traktoren. Zwei Drittel der in Deutschland produzierten Traktoren stammten aus Mannheimer Fertigung. Das Produktionsprogramm für den weltweiten Markt umfasst 21 verschiedene Grundmodelle von 90 PS bis 250 PS in unterschiedlichen Versionen und zahlreichen Ausstattungsvarianten.

Im APRODI-Projekt verfolgt das John Deere Werk Mannheim unter anderem die Weiterentwicklung eines digitalen Shopfloor Managements und die Weiterentwicklung der im Unternehmen seit 1992 erfolgreich etablierten Gruppenarbeit. Alle Montagegruppen arbeiten relativ selbstorganisiert, sind durch Ziele gesteuert und managen ihren Bandabschnitt von der Personalplanung bis zum KVP selbst. Zum Management der Gruppenarbeit gehört das jeden Morgen stattfindende und etwa 15 min dauernde Shopfloor-Meeting.

Während der Projektlaufzeit hatte ein interdisziplinäres Team von Mitarbeiterinnen und Mitarbeitern aus verschiedenen Unternehmensbereichen ein digitales Shopfloor Management-System entwickelt, das sukzessive eingeführt wurde. Aktuelle Produktionsdaten und Kennzahlen wurden größtenteils nicht mehr manuell, sondern automatisch in einer Datenbank erfasst. Das morgendliche Shopfloor-Meeting fand vor einem touchfähigen Großbildschirm statt, an dem der Gruppensprecher einfache DatenVisualisierungen der für die Produktion und Montage relevanten Kennzahlen präsentierte sowie mit den Mitarbeitenden die Produktionsvorschau besprach und den KVP erörterte.

Im John Deere Werk Mannheim wurde erkannt, dass die Erfolgsbausteine des Shopfloor Managements Transparenz sowie Regeln, Standards, Verhaltensweisen und Methoden erfordern. Für die erfolgreiche Einführung und Anwendung eines digitalen Shopfloor Management-Systems ist es zudem erforderlich, digitale Kompetenzen im 
Unternehmen zu entwickeln, d. h. im Wesentlichen Lernkompetenz und Veränderungskompetenz zu vermitteln. Die Mitarbeitenden müssen bereit sein, sich neue Fähigkeiten anzueignen, und ein Bewusstsein entwickeln, dass sich Arbeitsweisen ändern. Zudem sollten die Auszubildenden auf die Shopfloor Management-Prozesse in der „Fabrik“ vorbereitet werden. Aus diesen Gründen hat das APRODI-Team im Zuge der Realisierungsphase ein Lernprojekt in der werkseigenen Ausbildungswerkstatt initiiert. Ziel des Lernprojekts war, den Auszubildenden des Werks frühzeitig das für die Gruppenarbeit essenzielle Shopfloor Management sowie den Umgang mit der digitalen Variante des Systems zu vermitteln. Mittels des vom ifaa - Institut für angewandte Arbeitswissenschaft entwickelten Handlungsleitfadens „Shopfloor-Management“ [2] wurde ein Workshop-Konzept angewendet, in dem ausgewählte Auszubildende aus sämtlichen Ausbildungsberufen des Werks die Arbeitsprinzipien und das Rollenverständnis des Shopfloor Managements kennenlernten, aber auch direkt die wesentlichen Erfolgsbausteine, wie u. a. visuelles Management, Kennzahlen, Regelkommunikation, systematische Problemlösung, für ihr eigenes Shopfloor Management-System erarbeiten konnten.

Ziel ist es, für jeden Ausbildungsberuf ein auf den Arbeitsprinzipien des Shopfloor Managements basierendes Arbeitssystem zu etablieren. Zukünftige Probleme sollen in den operativen Prozessen und Aufgaben der Ausbildungswerkstatt zielorientiert in einem Team, bestehend aus Auszubildenden und Ausbildungsleitern, behoben werden. In Shopfloor-Meeting ähnlichen Gruppenbesprechungen sollen wöchentlich die Prozesse bezüglich unterschiedlicher Kriterien wie z. B. Sicherheit, Ordnung, Effizienz diskutiert, Probleme aufgedeckt und Handlungspläne festgelegt werden.

\subsubsection{Für Nachhaltigkeit im operativen Geschäft sorgen: Die Stabilisierungsphase am Beispiel der ZF Friedrichshafen AG, Schweinfurt}

Die Stabilisierungsphase markiert idealerweise den Übergang vom Projekt ins laufende „operative Geschäft", für das eine besondere Projektorganisation nicht mehr erforderlich ist. Nötig oder zumindest wünschenswert ist es jetzt, einen Projektabschluss zu gestalten, der Zeit und Raum für Ergebnispräsentation, Abnahme, Auswertung, Reflexion und schließlich „Übergabe“ an die operativ Verantwortlichen ermöglicht. In der betrieblichen Wirklichkeit häufen sich gerade im letzten Drittel des Veränderungsprozesses Problemkonstellationen, die auch schon in der Realisierungsphase auftauchen können, und die es zu lösen gilt:

- Managemententscheidungen für notwendige Neu- und Umplanungen verzögern sich.

- Teilprojekte agieren ohne die eigentlich wünschenswerte Abstimmung.

- Der Austausch der Projektakteure und Zwischenbilanzen kommen zu kurz.

- Tragende Personen scheiden aus oder wechseln in eine neue Position bzw. Rolle. 
Hinzu treten nicht selten weitere Herausforderungen:

- Ziele rauf, Ressourcen runter! Wie geht man unter diesem operativen Druck mit den noch offenen Punkten und „losen Enden“ aus dem Betriebsprojekt um?

- Wie gelingt es, einen guten Projektabschluss zu finden, dabei genügend Raum für „Lessons learned“ zu schaffen, die Aufmerksamkeit des Managements zu erhalten und Ressourcen für die Weiterführung im Alltag zu bekommen?

Bei ZF Friedrichshafen in Schweinfurt wurden im Hinblick auf diese Herausforderungen der Verstetigung und Stabilisierung folgende Ansätze verfolgt: Das APRODIBetriebsprojekt stand von Beginn an unter hohem Erwartungsdruck und es erfreute sich nach der erfolgreichen Etablierung der Projektstrukturen in der Orientierungsphase auch einer hohen Managementaufmerksamkeit. Vor diesem Hintergrund ergab sich nicht erst in der Stabilisierungs- sondern bereits in der Realisierungsphase die Herausforderung, Teilprojekte untereinander zu vernetzen, die Berichterstattung zu verstetigen sowie Zeit und Raum für Steuerkreistreffen als Ort der nötigen Managemententscheidungen zu schaffen.

Über die Vernetzung durch das technische Assistenzsystem (Instandhaltungsplanungssystem) hinaus führten sogenannte Vernetzungsforen die Menschen, aber auch die von ihnen im betrieblichen Alltag erzeugten Ergebnisse und Lösungen stärker zusammen. Zur Verstetigung und damit zur Stabilisierung des Projektvorgehens haben (meist in Verbindung mit den Vernetzungsforen) Meilenstein-Meetings im Projektteam und anschließende Steuerkreis-Treffen beigetragen. So konnte das Projektteam seine Vorschläge direkt im Hinblick auf die anschließende Vorstellung im Steuerkreis ausarbeiten, und der Steuerkreis konnte anschließend gut informierte Entscheidungen treffen. Eine Projektteamklausur, wie sie schon zur Vorbereitung des Fokusentscheids erfolgreich war, lieferte zu schon vorhandenen Aufgaben und Aktivitäten des Ersatzteilmanagements weitere strategische Initiativen.

\subsection{Erkenntnisse und Botschaften}

Die Ansätze für zu begleitende Digitalisierungsprozesse stellten sich im APRODIProjekt sehr vielfältig dar. Sie umfassten die Neuentwicklung komplexer technischer Lösungen, die Optimierung und Integration bereits bestehender Systeme bis hin zur strategischen Zielerarbeitung und der Konzeption von Prozessen zum Kompetenzaufbau. Vorgehensweisen und Methodeneinsatz waren deshalb an den jeweils vorfindlichen Entwicklungsständen der Unternehmen(sbereiche) auszurichten und an deren spezifische Bedarfe anzupassen. Entstanden ist dadurch eine variantenreiche Toolbox, die es den Nutzenden aber gleichfalls ermöglicht, die Instrumente auszuwählen, die sich an ihren individuellen Belangen orientieren. 
Der ganzheitliche Blick auf das Unternehmen - auf Technik, Organisation und Mensch - und damit auch auf die ihm innewohnende Kultur galt als wichtiger ARPODIGrundsatz. Gerade in Konzernstrukturen kam es auch darauf an, möglichst früh die internen Regeln und damit beispielsweise die Grenzen der Entscheidungsfreiheit zu kennen und richtig einschätzen zu können. Das zugrunde liegende Prozessverständnis mit Reflexion und rollierender Planung hat sich bewährt, um auf wechselnde betriebliche Bedarfe und Bedingungen eingehen zu können.

Eine wichtige Erfahrung bestand zudem darin, dass technologiefokussierte digitale Innovationen nur dann einen Mehrwert bringen können, wenn die sie umgebende Prozesslandschaft gut strukturiert ist. Das bedeutet, dass bestehende Prozesse häufig erst auf „Vordermann“ gebracht werden müssen, bevor die technische Lösung eingesetzt wird. Auch gilt es, bestehende gute Praxis in den Betrieben zu entwickeln und weiterzuführen. Eine soziotechnische Orientierung kann dabei hilfreich sein, um die organisatorische Praxis zu bearbeiten. Dies haben die APRODI-Betriebspartner individuell bewältigt. In der praktischen Projektarbeit ergab sich daraus die große Herausforderung, einerseits möglichst alle betroffenen Prozesse im Auge zu behalten, alle Schlüsselpersonen einzubeziehen und andererseits die Aufgaben bearbeitbar zu gestalten und den laufenden Betrieb nicht zu gefährden. Dabei sind Regularien und Standards (der IT-Nutzung) und Kapazitätsfragen (insbesondere bei IT und KnowhowTrägern) zu klären.

Schließlich ist der erweiterte Blick auf Arbeitsaufgaben, Abläufe und die Anforderungen aus Beschäftigtensicht ein zentraler erfolgskritischer Faktor, wenn es um die Gestaltung technischer Systeme geht. In APRODI erwies sich daher das Heranziehen soziotechnischer Ansätze und Kriterien zur nutzergerechten Gestaltung als unabdingbar.

\subsection{Offene Forschungsfragen}

Während der Entwicklungsarbeiten tauchte immer wieder die Frage nach dem Stellenwert einer Strategie auf: Braucht es eine Digitalisierungsstrategie? Oder eher eine Unternehmens-/Standort-Strategie mit Digitalisierungsaspekten? Bei der Bearbeitung dieser Frage wurde in den fünf Betriebsbeispielen auf unterschiedliche Vorgehensweisen zurückgegriffen. Während sich beispielsweise Continental Teves zunächst intensiv der strategischen Ausrichtung des Werks widmete, waren die Entwicklungsschritte in den anderen Unternehmen stärker von der praktischen Umsetzung und der Lösung eines Problems getrieben. Auf die Frage, wie viel und welche Strategie es braucht konnte in APRODI keine eindeutige Antwort gegeben werden, sie bleibt daher als offene Forschungsfrage bestehen.

Während der partizipative Ansatz zur Beteiligung von Beschäftigten und Führungskräften in allen Betrieben zu einem erfolgreichen Entwickeln und Umsetzen von Maßnahmen geführt hat, stand der Mitbestimmungsprozess aus verschiedenen 
Gründen nicht im Vordergrund der APRODI-Betriebsprojekte. Dabei sind gerade bei Digitalisierungsprojekten, die sich häufig in einem Experimentierraum bewegen, Fragen der Zusammenarbeit zwischen Betriebsrat und Arbeitgebervertreter sowie der jeweiligen Erwartungshaltung von elementarer Bedeutung für den Erfolg. Digitalisierungsprojekte folgen jedoch einer neuen Dynamik, auf die traditionelle Mitbestimmungsstrukturen nur schwer eine Antwort finden können. Hier lohnt es, im sozialpartnerschaftlichen Dialog künftig verstärkt zu forschen.

\subsection{Produkte und Angebote}

Die Erkenntnisse aus dem Projekt, empfehlenswerte Vorgehensweisen und Instrumente zur Gestaltung von betrieblichen Digitalisierungsprozessen stehen in einer interaktiven Toolbox für andere Unternehmen zur Verfügung. Diese eröffnet multimedial Zugang zu den eingesetzten Werkzeugen. O-Töne und Beispiele veranschaulichen die Ansätze und Erfahrungen und bieten so auch kleineren Unternehmen Zugang zu arbeits- und prozessorientierter Digitalisierung.

Daneben bietet die Praxisbroschüre „Arbeits- und prozessorientiert digitalisieren“ [7] eine umfassende Übersicht über Vorgehen, Erfahrungen und Methoden, die in den betrieblichen Fallbeispielen während der verschiedenen Phasen eingesetzt wurden. Praktiker - innerhalb oder außerhalb eines Betriebs - finden mit der Broschüre Rüstzeug für strukturierte und zielführende Digitalisierungsprozesse.

Download unter www.aprodi-projekt.de

\section{Projektpartner und Aufgaben}

- Agfa-Gevaert HealthCare GmbH

Entwicklung digitaler Assistenzsysteme zur mitarbeiterorientierten Unterstützung komplexer Montageprozesse

- Continental Teves AG \& Co. oHG Digitalisierung begreifbar machen

- DuBay Polymer GmbH Optimierung einer Beteiligungskultur im Schichtbetrieb einer High Performance Arbeitskultur

- John Deere GmbH \& Co. KG, Werk Mannheim Digitales Shopfloor-Management bei Gruppenarbeit

- ZF Friedrichshafen AG, Standort Schweinfurt Unterstützung komplexer Instandhaltungsaufgaben

- GITTA Gesellschaft für interdisziplinäre Technikforschung Technologieberatung Arbeitsgestaltung $\mathbf{m b H}$

Entwicklung eines partizipativen Vorgehens zur kompetenzorientierten Gestaltung von Arbeitsorganisation, IT-Infrastruktur und Personaleinsatz 
- ifaa - Institut für angewandte Arbeitswissenschaft e. V.

Instrumentierung und transferfähige Aufbereitung des APRODI-Vorgehens

- Universität Duisburg-Essen, Institut Arbeit und Qualifikation (IAQ)

Konzepte beteiligungsorientierter soziotechnischer Gestaltung in Digitalisierungsprozessen produzierender Unternehmen

- Rationalisierungs- und Innovationszentrum der Deutschen Wirtschaft e. V. Kompetenzzentrum

Optimierung und Bewertung interner und externer Austauschprozesse zur Sicherung von Relevanz, Konsensfähigkeit und Anwendbarkeit der Entwicklungsergebnisse

\section{Literatur}

1. Bendel A, Latniak E (2020, i.V.) ,soziotechnisch - agil - lean“: Konzepte und Vorgehensweisen für Arbeits- und Organisationsgestaltung in Digitalisierungsprozessen. zur Veröffentlichung angenommen in der Zeitschrift „Gruppe.Interaktion.Organisation“

2. Conrad R, Eisele O, Lennings F, ifaa (Hrsg) (2019) Shopfloor-Management - Potenziale mit einfachen Mitteln erschließen: Erfolgreiche Einführung und Nutzung auch in kleinen und mittelständischen Unternehmen. Springer, Berlin, Heidelberg

3. Herrmann T, Nierhoff J (2019) Heuristik 4.0. Heuristiken zur Evaluation digitalisierter Arbeit bei Industrie-4.0 und KI-basierten Systemen aus soziotechnischer Perspektive. FGW-Studie. Hg. v. Hartmut Hirsch-Kreinsen und Anemarie Karačić. Düsseldorf (Digitalisierung von Arbeit, 16)

4. Lange K, Longmuß J (2015) 6.3 Das PaGIMO-Veränderungsmodell. Zink K, Kötter W, Longmuß J, Thul M (Hrsg) Veränderungsprozesse erfolgreich gestalten. 2., aktualisierte und erw. Aufl. Berlin: Springer Vieweg (VDI-Buch):169-173

5. Latniak E (2013) Leitideen der Rationalisierung und der demografische Wandel - Konzepte und Herausforderungen. In: Hentrich J, Latniak E (Hrsg) (2013) Rationalisierungsstrategien im demografischen Wandel: Handlungsfelder, Leitbilder und Lernprozesse. Springer Gabler, Wiesbaden, S $27-57$

6. Pasmore W, Winby S, Mohrman S, Vanasse R (2018) Reflections: sociotechnical systems design and organization change. J Change Manag 1-19. https://doi.org/10.1080/14697017.2018 .1553761

7. RKW Kompetenzzentrum (Hrsg) (2020) Arbeits- und prozessorientiert digitalisieren. Praxisbroschüre. RKW Kompetenzzentrum, Eschborn. Download unter: https://www.aprodi-projekt. de/ergebnisse/arbeits-und-prozessorientiert-digitalisieren/

8. Ulich E (2005) Arbeitspsychologie. 6., überarb. u. erw. Aufl. Zürich: vdf Hochschulverlag

9. Winby S, Mohrman S (2018) Digital sociotechnical system design. J Appl Behav Sci 54(4):399-423. https://doi.org/10.1177/0021886318781581 
Open Access Dieses Kapitel wird unter der Creative Commons Namensnennung 4.0 International Lizenz (http://creativecommons.org/licenses/by/4.0/deed.de) veröffentlicht, welche die Nutzung, Vervielfältigung, Bearbeitung, Verbreitung und Wiedergabe in jeglichem Medium und Format erlaubt, sofern Sie den/die ursprünglichen Autor(en) und die Quelle ordnungsgemäß nennen, einen Link zur Creative Commons Lizenz beifügen und angeben, ob Änderungen vorgenommen wurden.

Die in diesem Kapitel enthaltenen Bilder und sonstiges Drittmaterial unterliegen ebenfalls der genannten Creative Commons Lizenz, sofern sich aus der Abbildungslegende nichts anderes ergibt. Sofern das betreffende Material nicht unter der genannten Creative Commons Lizenz steht und die betreffende Handlung nicht nach gesetzlichen Vorschriften erlaubt ist, ist für die oben aufgeführten Weiterverwendungen des Materials die Einwilligung des jeweiligen Rechteinhabers einzuholen.

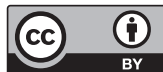

